\title{
Time-Resolved Digital Holography in the Investigation of Ablation and Micro Fabrication by Femtosecond Pulses
}

\author{
Nerijus ŠIAULYS, Andrius MELNINKAITIS, Aivaras URNIEŽIUS, Viačeslav KUDRIAŠOV and Valdas SIRUTKAITIS
}

\author{
Laser Research Center, Vilnius University, Sauletekio av. 10, LT-10223 Vilnius, Lithuania \\ E-mail: valdas.sirutkaitis@ff.vu.lt
}

\begin{abstract}
During the last years it was shown that time-resolved digital holography (TRDH), combining the conventional offaxis digital holography and ultrashort probing laser pulses results in versatile tool suitable for ultra-fast phenomena studies. The temporal resolution of this method depends on the duration of the used ultrashort probing pulses and measurements could be performed in temporal range from $\sim 20$ fs to $\sim 10 \mathrm{~ns}$. We review features of TRDH with femtosecond pulses and its applications for the investigation of such processes occurred or used in laser micro fabrication as femtosecond pulse filamentation in transparent solids, thin film damage by femtosecond pulses and one and double pulse femtosecond laser ablation.
\end{abstract}

DOI: $10.2961 / \mathrm{j} \operatorname{lmn} .2013 .03 .0006$

Keywords: time-resolved digital holography, femtosecond pulses, ablation, filamentation, damage

\section{Introduction}

The ability to excite matter with ultrashort light pulses and probe its subsequent evolution on the femtosecond time scale has opened up complete new realms of science. Nevertheless, in conventional pump-probe technique there is no possibility to obtain the spatial information of excited material region, however the understanding of spatiotemporal evolution of laser-matter interaction is of major importance for further development of ultrashort laser science and technology. In order to characterize non-uniform optical properties of the disturbed medium in the case of femtosecond pulse filamentation, damage and ablation various methods can be applied. Photography, shadowgraphy, interferometric- and holographic imaging are among the mostly used [1]. All methods have both weak and strong sides in respect to reveal small amplitude and phase inhomogeneities of the disturbed optical properties. The evolution of the disturbed medium or ablated material on the short temporal scale can be performed if these techniques are used together with temporally resolved methods, like shadowgraphic imaging [2], time resolved microscopy [3] or pulsed holography [4]. A quite prospective method is a digital holographic approach, enabling to record the whole wave information and then perform reconstruction of the object characteristics using numerical calculations [5]. Digital holography is capable to obtain quantitative amplitudeand phase-contrast images with good spatial resolution. During the last years it was shown that time-resolved digital holography (TRDH), combining the conventional offaxis digital holography and ultrashort probing laser pulses results in versatile tool suitable for ultra-fast phenomena studies. It has been already applied in several in-line $[6,7]$ and off-axis $[8,9]$ regimes for material studies. The temporal resolution of this method depends on the duration of the used ultrashort probing pulses and measurements could be performed in temporal range from $\sim 10$ fs to $\sim 10 \mathrm{~ns}$. Merits of off-axis TRDH: a) single-shot measurement with temporal resolution equal to used laser pulse duration, b) spatial resolution better than $2 \mu \mathrm{m}$ using magnifying optics, c) phase-contrast images are quantitative - no calibration required, d) differential phase-contrast imaging (with and without pump pulse), e) phase sensitivity down to $\sim \lambda / 500$ can be achieved, f) elimination of incoherent radiation via digital spatial filtering, j) digital focusing of the image.

We review futures of TRDH with femtosecond pulses and its applications for the investigation of such processes occurred or used in laser micro fabrication as femtosecond pulse filamentation in transparent solids, thin film damage by femtosecond pulses and one and double pulse femtosecond laser ablation. The off-axis hologram recording geometry was used in our experiments in order to separate the twin-image and so-called DC term of diffraction by means of simple digital spatial filtering.

\section{Experimental setups for TRDH}

The optical scheme of the experimental setup in which temporal resolution is equal to the pulse duration of the used laser is shown in Fig. 1. The light pulses of $300 \mathrm{fs}$ duration from high repetition rate ytterbium laser operating at $1030 \mathrm{~nm}$ wavelength ("Pharos", Light Conversion) were used as a light source. This type of femtosecond lasers is the most promising for industrial micro machining applications. In the setup the initial pulse was divided in two, going into two different channels, namely pump and probe. The pump pulses remained unchanged and were used for initiation of the damage or ablation processes. The probe pulses were frequency-doubled to $515 \mathrm{~nm}$ wavelength and after passing the delay line were launched into the MachZehnder interferometer comprising the holographic setup. The delay line was able to provide time intervals up to several ns between the pump and probe pulses.

Holographic recording scheme works as follows. The incoming probe pulse is divided into object and reference pulses at the first beamsplitter BS1, then passes two different arms with the same optical path, and after that recombine at the second beamsplitter BS2. The object arm (BS1M5-BS2) is made in a microscope configuration, using microscope objectives $(20 \mathrm{x}, 0.4 \mathrm{NA})$, that produce magni- 
fied image of the object at some distance beyond the interferometer (at CCD2 location). As the object pulse is propagating through the disturbed air due to the ablation it acquires spatial phase deformation corresponding to the change in optical density near the target surface. After recombination of the object and reference pulses at BS2, their interference pattern is recorded after some distance at the CCD1 plane. This is a hologram of the probe and reference pulse which corresponds to the following intensity distribution

$$
H(x, y, 0)=\left|U_{o}\right|^{2}+\left|U_{r}\right|^{2}+U_{o} U_{r}^{*}+U_{o}^{*} U_{r},(1)
$$

where $U_{o}$ and $U_{r}$ denote the complex fields of the object and reference pulses, respectively. Hologram is recorded in the off-axis configuration where the reference pulse after BS2 propagates with a small angle with respect to the object beam (see Fig.1). To sample the hologram correctly with the CCD, the fringe spacing in the interferogram (1) should be twice as large as the matrix pixel size in accordance with Nyquist sampling theorem [5].

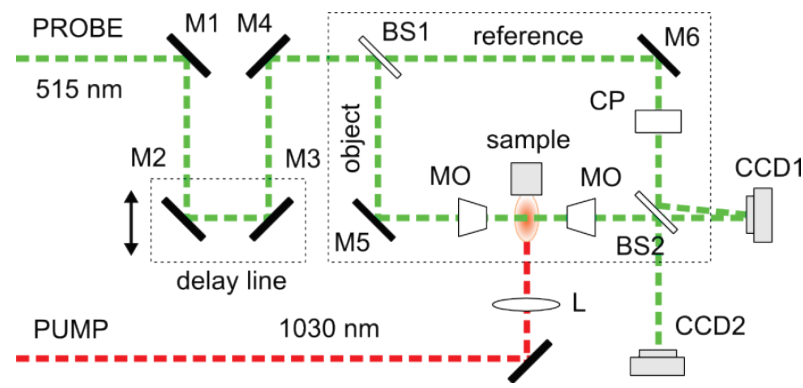

Fig. 1 (Color online) A part of off-axis TRDH setup used for the laser-induced ablation studies: M1-M6 - high reflection mirrors; $\mathrm{BS}-50 \%$ beam splitters, $\mathrm{MO}$ - microscope objectives; $\mathrm{CP}$ optical path compensation plate, $\mathrm{L}$ - focusing lens $(5 \mathrm{~cm}$ focal length); CCD1 - detector for hologram registration, CCD2 - detector for object image and plasma emission registration.

Having the hologram intensity distribution and the intensity of a reference beam, one can completely reconstruct the whole wave-front of the object field. Multiplying the hologram with the complex amplitude of the measured reference field the wave field just after the hologram becomes

$$
U(x, y, 0)=U_{r}\left(\left|U_{o}\right|^{2}+\left|U_{r}\right|^{2}\right)+U_{o}\left|U_{r}\right|^{2}+U_{o}^{*} U_{r}^{2} .
$$

This field then is numerically propagated by distance $d$ from the CCD1 plane to the imaging plane calculating the Huygens-Fresnel integral over the hologram area $S$

$$
U(x, y, d)=\frac{i}{\lambda} \iint_{S} U(x, y, 0) \frac{e^{-i k r}}{r} d x d y .
$$

Within the paraxial (Fresnel) approximation integral (3) may be easily calculated using the convolution approach [5]. Calculation gives the diffracted magnified field at the image plane located at distance $d$ after the CCD1. Actually, the calculation gives three different fields, in accordance with (2), but only the third term corresponds to the incident object wave and is useful. In the numerical reconstruction these fields can be well separated, when the off-axis holo- graphic configuration is used during the hologram recording [5].

Note also, that the object probe field acquires not only the amplitude and phase changes corresponding to the process under investigation. Propagation in the object arm even through the undisturbed media induces aberrations due to the lens imperfections and other systemic wavefront aberrations [10]. This wavefront modification adds to the useful changes each time the measurement is performed. After the reconstruction this systemic change has to be subtracted from the complex object field and it allows get the complex amplitude distribution corresponding only to the changes in the material.

Thus, experimentally we have to make a registration of several types of images taken at particular time delay value: a) bias-dark image to eliminate unavoidable camera noise and ambient light; b) intensity distribution of the reference pulse; c) hologram of the object wave when a pump pulse is off; d) hologram of the object wave when laser pulse is on. All experiments are performed after just a single laser shot. Thus for each probe delay value one records a hologram corresponding to a time moment of a dynamic process. All reconstruction steps are performed numerically using a personal computer.

Moreover, the setup had additional camera to record amplitude information. A shadowgraphic image of the ablation plume can be recorded with a CCD2 placed exactly at the imaging plane of the holographic microscope. Plasma emission can also be registered with CCD2 but only when the probe pulse is switched off, as the plasma emission is a rather weak incoherent light.

From the reconstructed USAF-1951 etalon images we determined that the holographic microscope has spatial resolution better than $2 \mu \mathrm{m}$. The temporal resolution of this setup coincides with the duration of the probe pulse used for pump-probe measurements which was equal to $300 \mathrm{fs}$.

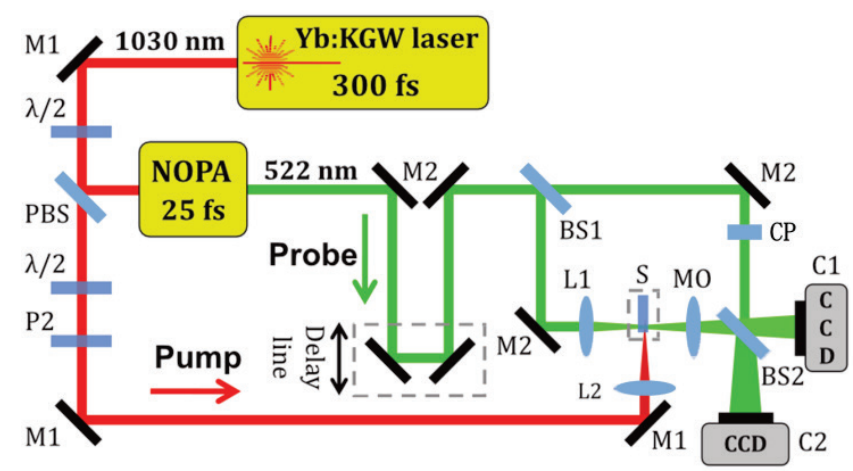

Fig. 2 (Color online) Experimental setup for TRDH experiments of filamentation in transparent materials with temporal resolution 25 fs.

For the studies of pulse transformation and free-carrier generation dynamics associated with the nonlinear propagation of femtosecond pulses in transparent materials we used TRDH setup with $\sim 25$ fs temporal resolution. The sketch of experimental setup is shown in Fig. 2. The same femtosecond pump laser with 300 fs pulses at central wavelength of $1030 \mathrm{~nm}$ as described in Fig. 1 was used. The 
incoming pulses were split into the pump and probe branches using $\lambda 2$ plate and polarizing beam splitter PBS. The pump pulses of fundamental wavelength were directed by high reflection mirrors M1 and focused by lens L2 onto the edge of the transparent sample (denoted $\mathrm{S}$ in the figure). The fluence level of the pump pulses was adjusted by $\lambda 2$ plate and polarizer P2. Probe pulses were produced by amplifying and compressing spectrally broadened supercontinuum pulses using noncollinear optical parametric amplification (NOPA). The NOPA setup was based on BBO (Type I) nonlinear crystal pumped by the third harmonic of the fundamental pulse. After the amplification and the prism-based compression, bandwidth limited probe pulses of $522 \mathrm{~nm}$ central wavelength and 25 fs duration were produced. The pulses from NOPA were directed through the delay line and further used for probing the optically induced changes in transparent materials within the modified Mach-Zehnder interferometer consisting of 50\% reflective beamsplitters BS1, BS2 and two high reflection mirrors M2. Focused to the sample and transmitted probe light was collected by a microscope objective MO $(20 \times, \mathrm{NA}=0.4)$ and directed to $\mathrm{CCD}$ cameras $\mathrm{C} 1$ and $\mathrm{C} 2$ both having $1280 \times$ 960 pixels of $3.75 \mu \mathrm{m}$ size. Camera $\mathrm{C} 2$ positioned in the image plane registered a magnified shadowgraphic image of the sample while camera $\mathrm{C} 1$ placed at some distance before the image plane captured the interference pattern (hologram) of the overlapped object and reference pulses.

\section{Applications of TRDH for investigation of filamen- tation in transparent materials}

Among the main advantages of TRDH technique is its ability to be operated in single shot mode and thus capturing whole trace of induced material changes of propagating light filament. The most popular pump probe geometry in this case is illustrated in Fig. 3. In this case long (300 fs) laser pulse enters the material with high energy that induces nonlinear changes in the material while very short $(<25$ fs) probe pulse is introduced sideways and thus takes a shadow of the filament with phase and amplitude information. Such approach requires special sample preparation as it should have at least four polished optical surfaces and the usage of the prechirped in the prism compressor probe pulses in order to reach minimal pulse duration at the plane where sample is located after passing first objective.

To illustrate this application we experimented with edge polished sapphire wafer. An typical outcome of such experiment is provided on Fig. 4 where amplitude (A) and phase (B) contrast images are taken at two different pump pulse energies. As can be seen filamentation is a result of dynamic process: competition between Kerr lens selffocusing and defocusing by diffraction and free electrons generated via multi-photon absorption and avalanche ionization [11-13]. At the beginning of the filament the Kerr effect is clearly visible that induces positive refractive index change: $\Delta n_{\mathrm{K}}=n_{2} \times I(>0)$, where $I$ is intensity and $\mathrm{n}_{2}$ is nonlinear refractive index (Kerr) coefficient. Promptly after that a plasma channel is formed. This channel is visible both in amplitude and phase contrast view. It leads to the negative refractive index change: $\Delta n_{\mathrm{p}}(<0)$ and reduced transmission due to free carrier absorption. As the energy increases pulses break into the multi filaments interacting with each other during the propagation.

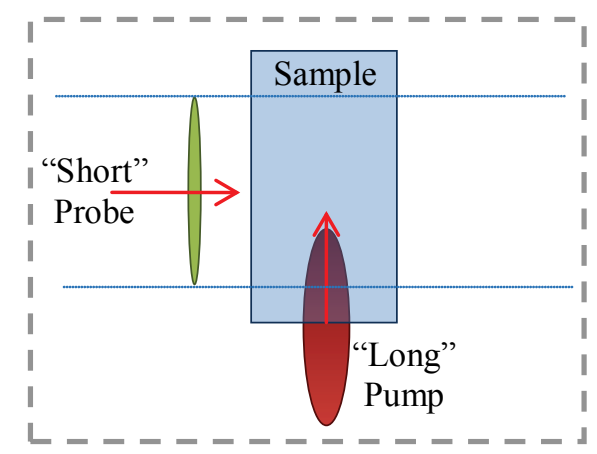

Fig. 3 (Color online) Geometry of pump-probe experiments for filamentation studies.
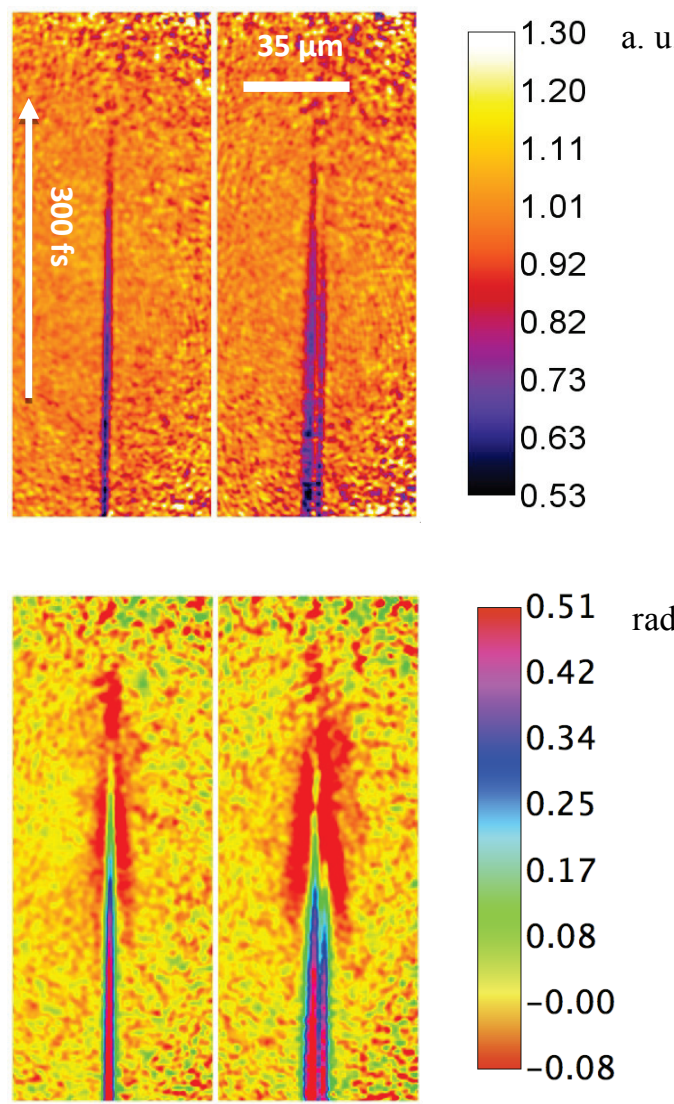

$\operatorname{rad}$

(B)

$$
4.9 \mu \mathrm{J} \quad 14 \mu \mathrm{J}
$$

Fig. 4 (Color online) Light induced filaments in sapphire - amplitude (A) and phase (B) view.

\section{Application of TRDH for optical breakdown imag- ing in thin films}

Next important application of TRDH method is the monitoring of ultrafast laser excitation and damage processes in thin films. Two kinds of geometries have been successfully demonstrated on thin films at Laser Research Center of Vilnius University. In both cases coating must be deposited on a transparent (for probe) substrate. In the first case sample was irradiated in off axis geometry (angle of incidence $45 \mathrm{deg}$ ) while probing pulse was propagating at 
normal incidence (Fig. 5). This approach allows capturing of time resolved optical response in whole optical (coating - substrate) system. By doing so it is possible to decompose all damage process into the series of space, amplitude and phase resolved sequence of images in time. This is a multi-shot experiment and thus requires a shifting of the sample after each damaging laser shot.

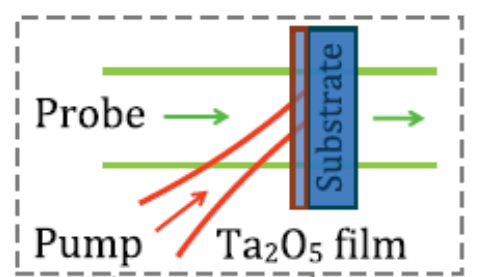

Fig. 5 (Color online) Pumping geometry of the coating: off-axis.

This geometry was firstly tested on tantala films deposited on fused silica. A typical example of optical response in coating when it is irradiated by fluence slightly above damage threshold $\left(1.72 \mathrm{~J} / \mathrm{cm}^{2}\right)$ can be illustrated by four different processes (Fig. 6). These can be split in three stages overlapped in time: Kerr effect ( $0 \mathrm{fs})$, generation of free electrons (700 fs), lattice heating trough electron relaxation and shock wave generation (1 ns).

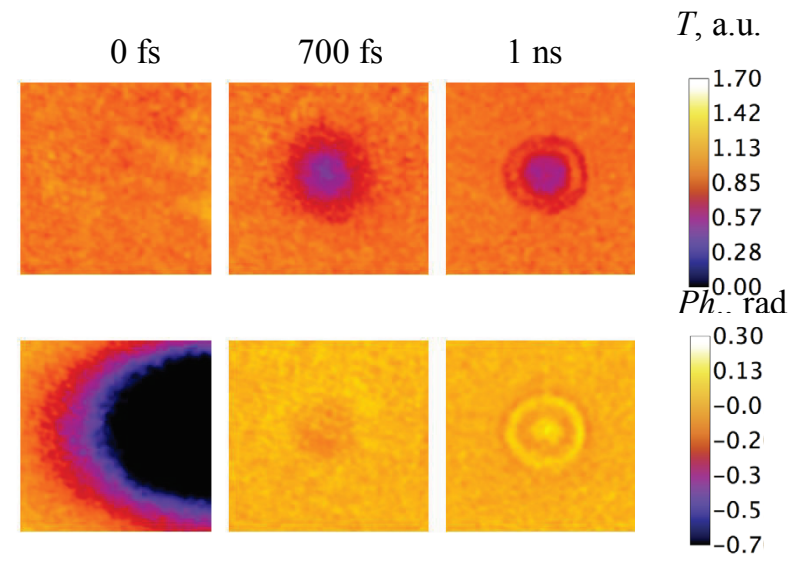

Fig. 6 (Color online) Amplitude (top) and Phase (bottom) contrast images taken in tantala coating at time delays representing different processes.

Another way to study early laser-matter interaction in films is similar to that used for filament studies in bulk. In this case TRDH technique is used when excitation of coating is done by pumping sideways (Fig. 7). This approach is more constrained by sample preparation requirements: deposited sample edge needs to be flattened by performing cutting and polishing steps. By polishing appropriate optical quality must be reached without contamination of coating. Nevertheless it is well suited in cases when single layers have sufficient thickness to couple sharply focused laser beam. The advantage of this pump-probe geometry is neglection of substrate properties: difference is taken between two (exited and non-exited coating) images. In this particular case pumping pulse does not propagate into substrate. Here the coating has sufficient propagation distance to estimate nonlinear refractive index and electron density. Here beam propagates trough the higher index coating acting as a waveguide.

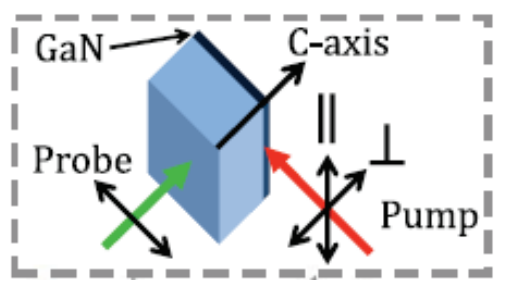

Fig. 7 (Color online) Pumping geometry of the coating: sideways.

This measurement is much faster if compared to previous one because of the fact that it is performed in single shot mode. As an example MOCVD grown GaN on sapphire [14] is taken. The characteristic optical response is illustrated in Fig. 8.

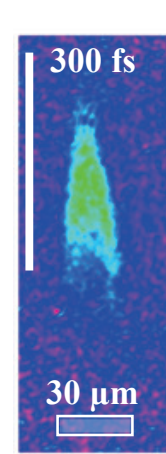

A a. u.

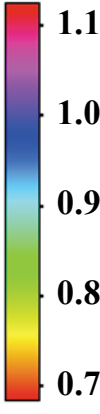

0.7

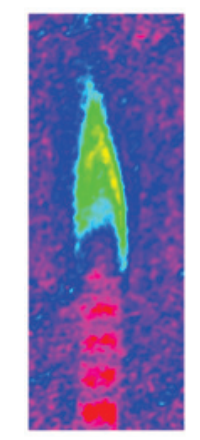

B
Fig. 8 (Color online) Amplitude (A) and phase (B) images taken in $20 \mu \mathrm{m}$ thick GaN layer: pump fluence at the entrance surface is $0.15 \mathrm{~J} / \mathrm{cm}^{2}$.

As can be seen in Fig. 8 the nonlinear dynamics of femtosecond pulse interaction in GaN crystalline film is observed with 25 fs temporal and higher than $2 \mu \mathrm{m}$ spatial resolution As in previous case of sapphire this technique allows a direct observation of nonlinear phenomena in the excited region: pulse self-focusing due to the Kerr effect and formation of free-electron plasma. Surprisingly plasma oscillations corresponding to terahertz frequency are also visible in phase contrast view. These effects cannot be distinguished in amplitude view providing the same information as shadowgraphic technique.

\section{Application of TRDH for laser ablation studies}

Ablation results in material ejection from the irradiated target surface after exceeding a certain fluence threshold $[15,16]$. A characteristic feature of this process is the formation of ablation plumes and the accompanying hydrodynamic compression of the ambient gas [17], producing supersonically propagating shock waves. TRDH imaging was used as an experimental tool in order to detect the amplitude and phase variations of the ablation dynamics after a 
single pulse [9]. According to the obtained data the shock wave expansion from the tempered steel occurs at the rates in the range $\sim 18 \mathrm{~km} / \mathrm{s}$ in the short initial period ( $<2 \mathrm{~ns}$ ) after ablating pulse. Here we present new results on the investigation of the one and double pulse femtosecond laser ablation by TRDH.

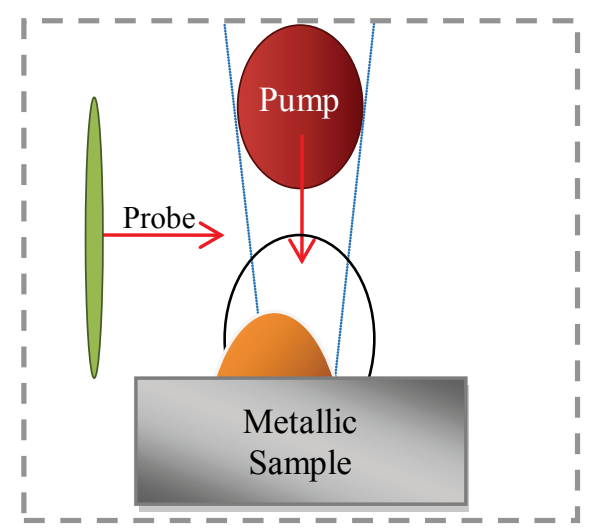

Fig. 9 Pump-probe geometry in ablation experiments used with TRDH.

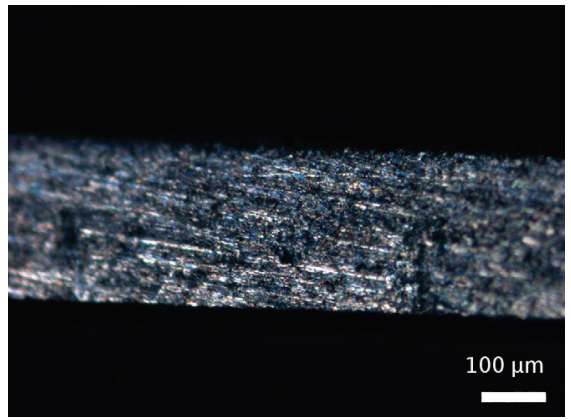

Fig. 10 Surface of the aluminium samples used in TRDH ablation experiments

For TRDH imaging of the ablation processes is used pump-probe geometry presented on Fig. 9. This is a multishot experiment and thus requires a shifting of the sample after each ablating laser shot. At the beginning we will discuss the limits in TRDH observation of ablation by femtosecond pulses. Taking into account that shock wave velocity is $\sim 18 \mathrm{~km} / \mathrm{s}$ the shock wave front propagates the distance equal to $2 \mu \mathrm{m}$ (such shift corresponds to the spatial resolution achieved in our TRDH setup) in $>100$ ps. So observable shift of the shock wave front could be registered only at probe pulse delays larger than $50 \mathrm{ps}$. So for the TRDH ablation experiments is used experimental setup in which temporal resolution is equal to the pulse duration (300 fs) of the used laser. Required spatial resolution is achieved using microscope objectives in the optical scheme and for the ablation experiments must be used thin (0.1-0.5 mm) polished metal samples as one presented on Fig. 10.

In our holographic ablation experiments different time delays (from 0.1 to $2.1 \mathrm{~ns}$ ) were used to investigate the temporal evolution of the shock wave and the ablation plume after a single and double pulse irradiation. A sharply focused pump beam at $1030 \mathrm{~nm}$ with at different fluencies with maximum reaching $\sim 19 \mathrm{~J} / \mathrm{cm}^{2}$ was used as a pump pulse. During the experiments the surface of the sample was perpendicular to the pump pulse and the probe pulse was collinear to the surface (Fig. 1). Shock wave forming near the target surface was registered from the side. For each single pulse ablation only fresh sites on the surface were used.

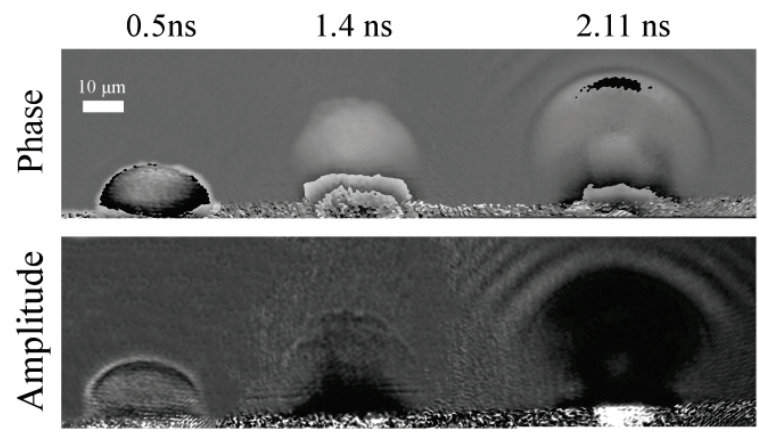

Fig. 11 Reconstructed phase- and amplitude-contrast images demonstrating the propagation dynamics of a shock wave at different pump-probe delay values after a single pulse. Fluence is $18.94 \mathrm{~J} / \mathrm{cm}^{2}$.

The ablation dynamics for a tempered steel sample is demonstrated in the reconstructed sequence of amplitudeand phase- contrast images (Fig. 11). They show the change in transmittance and phase change of the probe pulse that propagates through the disturbed air near the target. This ablation dynamic can be separated into two phases, namely shock wave formation and material expulsion from surface (ablation plume). The shock front appears as a thin dark line in the amplitude images, demonstrating an abrupt change of the refractive index inside the discontinuity layer. Such change is also replicated in the phase-contrast images, where the shock front exhibits as area with a positive phase shift. Positive phase shift means the increased optical density at the shock front, hence the probe pulse is delayed with respect to the ambient air acquiring a positive phase. The early shock wave appears as a slightly curved wave, which later transforms into a hemispherical blast wave. We have determined that shock wave appears near the surface after time delays of about $\sim 0.3 \mathrm{~ns}$. It means that the energy deposition to the material and the generation of a shock wave are completely separated in time. Subsequently when shockwave propagates away from the surface it loses its energy and the abrupt phase change at the shock front becomes less visible. This can be seen already at 1.4 ns delay.

Laser ablation with the same parameters was performed on four different metals. These experimental results show that these metals interact very similarly to the laser pulses. The main difference is the speed of the shock wave. The results are visible in the Table. 1.

Table 1. Shockwave speed at different fluence for different metals

\begin{tabular}{|l|l|l|l|}
\hline Metal & $6.5 \mathrm{~J} / \mathrm{cm}^{2}$ & $3 \mathrm{~J} / \mathrm{cm}^{2}$ & $1.5 \mathrm{~J} / \mathrm{cm}^{2}$ \\
\hline
\end{tabular}




\begin{tabular}{|l|l|l|l|}
\hline Steel & $11.9 \mathrm{~km} / \mathrm{s}$ & $10.5 \mathrm{~km} / \mathrm{s}$ & $8.49 \mathrm{~km} / \mathrm{s}$ \\
\hline Copper & $10 \mathrm{~km} / \mathrm{s}$ & $6.36 \mathrm{~km} / \mathrm{s}$ & $5.86 \mathrm{~km} / \mathrm{s}$ \\
\hline Nickel & $8.36 \mathrm{~km} / \mathrm{s}$ & $6.42 \mathrm{~km} / \mathrm{s}$ & $5.78 \mathrm{~km} / \mathrm{s}$ \\
\hline Aluminum & $10.9 \mathrm{~km} / \mathrm{s}$ & $7.12 \mathrm{~km} / \mathrm{s}$ & $5.45 \mathrm{~km} / \mathrm{s}$ \\
\hline
\end{tabular}

Similar experiments were performed by using double femtosecond pulses. In this particular case of laser ablation increase in the mass removal ratio was observed. The strongest effect was when both pulses were of identical energy density $10 \mathrm{~J} / \mathrm{cm}^{2}$ (Fig. 12). When the energy of delayed pulse is higher than that of first pulse, increase of the shock wave diameter was detected. Specific delay between double pulses is optimal for maximum ablation efficiency (in case of steel it is $\sim 40 \mathrm{ps}$ when both pulses are of the same energy).

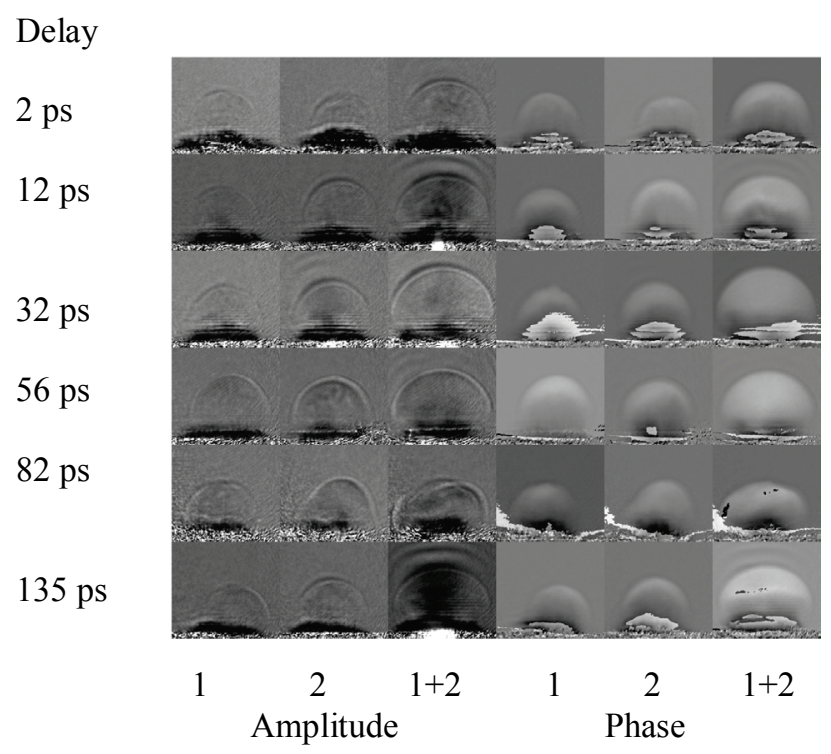

Fig. 12 Reconstructed amplitude and phase images in case of double pulse ablation at different delay between pulses. 1- corresponds to the first pulse ablation, 2- corresponds to the second pulse ablation, $1+2$ - corresponds to the double pulse ablation.

\section{Conclusions}

Overall, it is demonstrated that TRDH provides new possibilities for the investigation of complex spatiotemporal dynamical effects in such processes occurred or used in laser micro fabrication as femtosecond pulse filamentation in transparent solids, thin film damage by femtosecond pulses and one and double pulse femtosecond laser ablation.

\section{Acknowledgments}

Authors thank Laurent Gallais for the preparation of samples with tantala films and Tadas Malinauskas and Tomas Grinys for the preparation of GaN samples.

The authors thank the European Social Fund Agency for the support according to research grant No. VP1-3.1-SMM10-V-02-007 (Development and utilization of new generation industrial laser material processing using ultrashort pulse lasers for industrial applications).

\section{References}

[1] S. Amoruso, R. Bruzzese, N. Spinelli, R. Velotta, J. Phys. B, At. Mol. Opt. Phys. 32(14), 131 (1999).

[2] S. S. Harilal, G. V. Miloshevsky, P. K. Diwakar, N. L. LaHaye, and A. Hassanein, Phys. Plasmas 19, 083504 (2012).

[3] A. Mermillod-Blondin, C. Mauclair, J. Bonse, R. Stoian, E. Audouard, A.Rosenfeld, and I. V. Hertel, Rev. Sci. Inst. 82, 033703-033708 (2011).

[4] E. Amer, P. Gren and M. Sjodahl, J. Phys. D: Appl. Phys. 41, 215502 (2008).

[5] U. Schnars, W. Jueptner, Digital Holography (Springer, Dordrecht, 2005).

[6] D. G. Papazoglou and S. Tzortzakis, Appl. Phys. Lett. 93, 041120 (2008).

[7] G. Rodriguez, A. R. Valenzuela, B. Yellampalle, M. J. Schmitt, and K.- Y. Kim, J. Opt. Soc. Am. B 25, 19881997 (2008).

[8] T. Balciunas, A. Melninkaitis, G. Tamosauskas, and V. Sirutkaitis, Opt. Lett. 33, 58-60 (2008).

[9] A. Urniežius, N. Šiaulys, V. Kudriašov, V. Sirutkaitis, A. Melninkaitis, Appl. Phys. A 108, 343-349 (2012).

[10] E. Cuche, P. Marquet, and C. Depeursinge, Appl. Opt. 38, 6994-7001 (1999).

[11] S. Minardi, A. Gopal, M. Tatarakis, A. Couairon, G. Tamošauskas, R. Piskarskas, A. Dubietis, and P. Di Trapani, Opt. Lett. 33(1), 86-88 (2008).

[12] W. Liu, S. Petit, A. Becker, N. Ak"ozbek, C. M. Bowden, C. M., and Chin, S. L., Optics Commun. 202, 189-197 (2002).

[13]E. Gaizauskas, A. Dubietis, V. Kudriasov, V. Sirutkaitis, A. Couairon, D. Faccio, and P. Di Trapani,

[Self-focusing: Past and Present], vol. 114/2009, ch. On the Role of Conical Waves in Self-focusing and Filamentation of Femtosecond Pulses with Nonlinear Losses, 457-479, Springer Berlin / Heidelberg.

[14]N. Šiaulys, V. Kudriašov, T. Stanislauskas, T. Malinauskas, A. Urniežius, and A. Melninkaitis, Opt. Lett. 37, 4916-4918, 2012.

[15] C. Phipps, Laser Ablation and Its Applications, 1st edn. (Springer, Berlin, 2006).

[16]B. N. Chichkov, C. Momma, S. Nolte, F. von Alvensleben, and A. Tünnermann, Applied Physics A: Materials Science and Processing 63, 109-115 (1996).

[17] D. Bauerle, Laser Processing and Chemistry, 3rd rev. ed.(Springer, Berlin, 2011).

(Received: September 13, 2013, Accepted: November 13, 2013) 